\title{
Comparing adaptation of constrained and unconstrained movements in three dimensions
}

\author{
Christopher Scharver, James Patton, Robert Kenyon, Eric Kersten \\ Rehabilitation Institute of Chicago, Chicago, Illinois
}

\begin{abstract}
This paper describes targeted reaching experiments conducted using a new augmented reality system. Combining a large-workspace immersive virtual environment with physical force feedback, the system distorted subjects' movements using a viscous curl force field Following previous experiments using a different robot, half the subjects were constrained to horizontal, planar movements. The remaining subjects performed unconstrained movements throughout the 3D workspace. Examining after-effects as an indication of learning, we found that constrained subjects learned the force field. However, it was difficult to detect whether the unconstrained subjects learned forces of identical magnitude. Our results found that force fields strengths eliciting constrained 2D adaptation have difficulty exhibiting after-effects for unconstrained 3D movements. The increased motor variability for 3D reaching movements requires consideration for future experimental design.
\end{abstract}

\section{INTRODUCTION}

W ITH new applications in haptics, telerobotics, and robotic rehabilitation, important questions have arisen on how to optimally teach or relearn hand-eye tasks. One question is whether one can enhance the learning process by exploiting what is known about sensory-motor adaptation. One key focus is on how manipulating the error signal (the strongest information for correcting behaviors) might facilitate learning. This manipulation can easily be brought about either by manipulating the mechanical environment by creating robotic forces or by distorting the visual display. One limitation has been that the various aparati for studying movement-induced neural adaptation have been small and constrained (e.g., planar) to two dimensions. Here we outline the design and implementation of a new system that can facilitate large workspace movements in three dimensions, and present an initial motor learning experiment on healthy subjects that identifies some differences between planar and free movements.

Supported by NIDRR RERC H133E020724-03, The American Heart Association 0330411Z, NIH R24 HD39627, NIH 5 RO1 NS 35673, NIH F32HD08658, Whitaker RG010157, and the Falk Trust. For additional information see http://www.SMPP.northwestern.edu/robotLab/

C. Scharver is a Research Engineer in the Sensory Motor Performance Program, Rehabilitation Institute of Chicago, Chicago, IL 60611, USA c-scharveranorthwestern.edu

J. Patton is Director of the Robotics Laboratory at the Rehabilitation Institute of Chicago, Chicago, IL 60611, USA j-pattonenorthwestern.edu

R. Kenyon is an associate professor in the Department of Computer Science, University of Illinois, Chicago, IL 60607, USA kenyon@uic.edu

E. Kersten ... University of Wisconsin Biomedical Engineering Graduate Program, Madison, WI, USA e jkersten@wisc.edu

\section{A. Motor Learning}

Movement skill typically requires adaptation and neural representations. Arguably the most primal form of intelligence involves adaptation of sensory-motor relationships. We often consider movement tasks such as reaching for a cup to be trivial, yet most are quite challenging due to factors such as the coupled nonlinear nature of arm dynamics [1] long feedback delays [2], and large activation times for muscles [3]. Consequently, rapid movements must be pre-planned using an expectation or "neural representation" of the outcome that is typically acquired from experience [4]. Research has shown that these internal models can be altered by distorting sensory-motor relationships in a variety of ways. Force fields, or forces governed by position and velocity, such as those imposed by a weight, damping, or other vector relations, cause a dramatic change in the movement patterns. Adaptation to a handheld mass occurs within a single motion [5], while more complex loads can take hundreds of movements [6]. This adaptation process is most evident when forces are unexpectedly removed, revealing after-effects. After-effects demonstrate that subjects have learned a neural representation of the force field, rather than simply "stiffening" their system to reject the disturbances [7]. It is important to note that both the adaptation and after-effects occur implicitly with minimal conscious attention to any goal, and hence can be used as a new tool for teaching movements. We have shown this to be true using forces that are applied in training [8], [9]. Previous experiments in our laboratory have been conducted using a planar manipulandum. Fig. 1 illustrates a subject with the planar manipulandum used for previous experiments.

\section{B. The importance of a capable visual display}

While the above research focuses on adaptation to a distortion in the mechanical world (kinetics), researchers have also observed similar adaptation to a more easily implemented visuomotor distortion (kinematics). These distortions involve exotic transformations using prisms [10], nonlinear mappings [11], or simple rotations or stretches [12], [13]. All of these distortions appear to cause the adaptive process. They can even trigger a rapid recovery from sensory disorders such as hemispatial neglect seen in stroke patients [14], which shortened the recovery process from months to hours. Tong and colleagues observed interference when both distortions depended on the same variables, suggesting that adaptation caused by these different sensory systems involve the same neural resources [15]. Our recent work [16] shows how a 


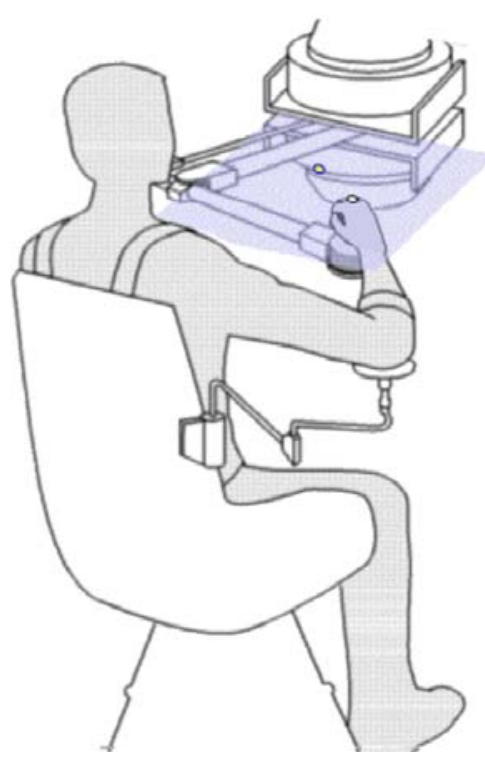

Fig. 1. Illustration of a subject interacting with the planar manipulandum robot. The device constrains all movements to a horizontal plane.

sensory system should facilitate the other, and a combination may be the most powerful. Clearly, visual-adaptive training is a very promising form of rehabilitation in brain-injured individuals.

Virtual display environments provide 3D user-interactive workspaces and allow a high level of dynamic flexibility. Integrating a force feedback robotic device, such a system communicates both visual and haptic feedback to subjects. The combination of physical and virtual feedback is termed augmented reality (AR). As defined by [17], augmented reality systems combine real and virtual information, allow real-time interactivity, and manage $3 \mathrm{D}$ registration. Such systems can provide significant features for rehabilitation [18]. First, these systems can present challenging environments while maintaining safety. Second, the computer integration facilitates data collection and analysis throughout the entire course of experiments. The environment captures data constantly, and that data may be analyzed afters experiments have concluded. Finally, the virtual environments can recreate functional tasks. Task-specific training is especially beneficial for recovery [19]. The virtual environment can create for the subjects a sense of actual presence within the virtual world.

In summary, there is a clear need for a capable testbed for scientific study on upper-extremity movement and rehabilitation that goes beyond what has currently been available. Robotic devices, designed to interface with humans, have already led to great strides in both fundamental and clinical research on the sensory motor system. The programming flexibility of these devices allows for a variety of scientific questions to be answered in psychology, neurophysiology, rehabilitation, haptics, and automatic control. Recently, we have begun developing and combining several state-of-theart devices that allow humans to visualize and feel synthetic objects superimposed on the real world for the purposes of rehabilitation. This paper's goal is to outline our motivations, present the design, describe our progress and future vision, and present early results that demonstrate its efficacy and identify the differences between the simpler application of two dimensional constrained movements and the more functional free movements in three dimensional space.

\section{DEVELOPMENT OF A LARGE WORKSPACE, HAPTIC/GRAPHIC SYSTEM}

Researchers conducted these experiments on the Virtual Reality Robotics and Optical Operation Machine (VRROOM). VRROOM utilizes a Personal Augmented Reality Immersive System (PARIS) display for rendering the virtual environment to subjects, and those images are super-imposed over a workspace containing a force feedback generating robot.

PARIS was designed within the at the University of Illinois at Chicago tested PARIS within the CAVE [20]. PARIS incorporates significant improvements over previous projection-based virtual reality displays. Previous systems such as the CAVE and the ImmersaDesk support stereovision, managing separate stereo images for each eye and tracking head motion. There remain at least two important depth perception cues, occlusion and accommodation, that are not supported correctly in previous displays. For instance, in a conventional projection-based virtual reality display, an object in front of the hand is obstructed by the hand itself. This occlusion causes a visual conflict because the hand, which should be behind the object, appears in front of the object. The object should be visible in front of the hand. The half-silvered mirror in the PARIS display superimposes the displayed image over the hands. One does not occlude the other. The second depth cue, accommodation, refers to muscles controlling the eye to adjust sharpness. In a conventional VR display the eye will always focus on the display screen, which is typically significantly farther than arm's reach. PARIS is designed so that the hands and the virtual object are the same distance as the image of the screen. The large workspace is particularly important for allowing the large arm movements important for upper-limb rehabilitation.

As shown in Fig. 2, a subject views graphics at arm's length superimposed over his hands. The half-silvered mirror allows viewing both the virtual environment and physical objects under the screen. By coordinating the display with a haptics device, PARIS enhances the subject's experience by providing the ability to both see and feel the virtual world.

The PHANToM 3.0 from SensAble Technologies [21] has a workspace measuring 900x900x300 mm, providing a significantly larger workspace from the PHANToM Desktop model used with the original PARIS. Arm movements from the elbow and shoulder require a larger work volume than the original 160x120x120 mm. The larger PHANToM's workspace is required for investigating upper-limb movements.

As with previous work on PARIS [22], the software relies on three key libraries. First, the CAVELib [23] creates the 


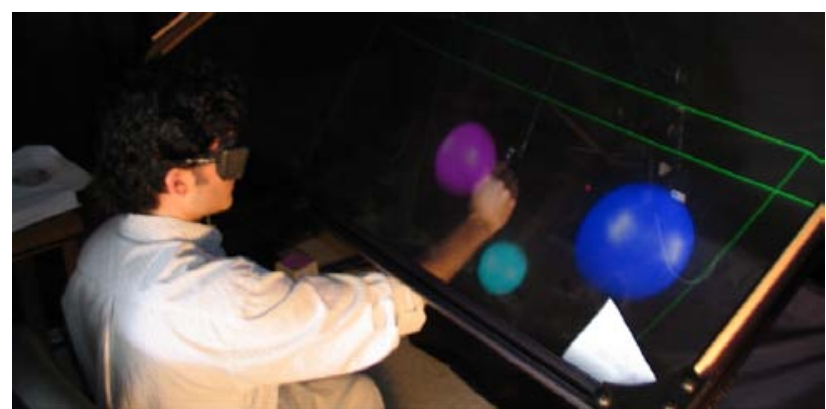

Fig. 2. A subject seated at VRROOM sees and feels objects in the virtual environment, but his hands are still visible.

stereoscopic OpenGL windows and manages a magnetic tracking system. Second, the Open Inventor scene graph [24] manages the graphics environment and renders it to the window. The scene graph simplifies programming and provides a structural organization for creating the graphics representations. Third and finally, GHOST [25] communicates with the PHANToM and controls its haptic servo loop. The entire system runs on a single Microsoft Windows XP workstation containing dual $2.2 \mathrm{GHz}$ Xeon processors and a NVidia Quadro 900XGL graphics card.

\section{EXPERIMENTAL METHODS}

Research devices have had several limitations that made it difficult to explore movements that span the full range functional activities. Three-dimensional (3D) movements are not only unrestricted, they must contend with the new challenge of gravitational effects that could reduce (or perhaps heighten) the potential of adaptive training. Before proceeding to applications such as neruo-rehabilitation, the experiment described below tested adaptation on healthy individuals. The VRROOM system presented a series of targets to research subjects, and it generated a force field to distort subjects' movements while reaching toward targets.

\section{A. Experiment Protocol}

Experiments placed ten subjects at VRROOM. None had prior experience with immersive environments. Ten subjects, randomly assigned to one of two groups (constrained 2D and unconstrained 3D). The first group practiced with a horizontally-aligned planar constraint on the handle, so that they were forced to move along a horizontal plane. This planar constraint created an interaction similar to previous experiments using the manipulandum, with the key difference being the virtual environment. These constrained subjects performed movements similar to the pilot-study [9] movements depicted in Fig. 3. The other five subjects practiced 3D targets and allowed unconstrained movement throughout the workspace.

Both groups of subjects reached toward four different targets. The planar-constrained targets were positioned within a horizontal plane, while the unconstrained 3D targets formed the vertices of a tetrahedron in such a way that the horizontal components of $3 \mathrm{D}$ targets lied on top of the paths of the $2 \mathrm{D}$

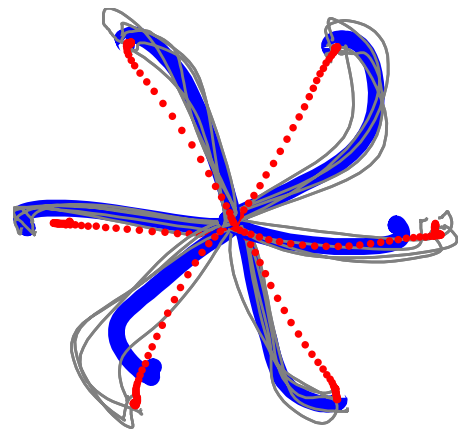

Fig. 3. Pilot data for constrained planar movement using a $20 \mathrm{~N}$ viscous curl field. The red dots indicate baseline trajectories, while the blue lines demonstrate after-effects. The grey lines represent the final phase, washout movements. This figure was originally published in EMBS 2004 [9].

target paths. In other words, the paths were aligned if viewed from above. The constrained subjects were presented a visual tabletop representing the constraint, and experimenters instructed subjects to not push against the planar constraint.

The experiment presented to subjects a series of spherical targets. Subjects moved to a starting position, and the destination target appeared in the virtual environment 15 $\mathrm{cm}$ away from the start. Once the cursor was within the target, the subject had to remain in place for one second. The application then presented a new target to the subject. The subjects were instructed to move 'rapidly to the target' and keep their movement speeds consistent throughout the experiment, but they could initiate movements at their own pace. Subjects were required to remain within a given radius of the target for one and a half seconds. After successfully reaching the target, each subject moved back to the center to prepare for a new movement.

VRROOM distorted subjects' movements by generating a viscous curl field. The current velocity factored into the force field calculations. Constrained subjects received the field's effects within their horizontal plane of movement, while unconstrained subjects received the full 3D effect. Equation 1 is the force calculation applied to subjects' movements. $F$ is the force in Newtons applied to the PHANToM's end effector, and $v$ is the current end effector velocity.

$$
=\left[\begin{array}{ccc}
0 & -25 & 25 \\
25 & 0 & -25 \\
-25 & 25 & 0
\end{array}\right] \text {. }
$$

Subjects performed 1507 movements broken into nine separate phases. Each trial included a phase-specific movement followed by a return to center movement:

- familiarization, 40 trials

- baseline (unperturbed), 20 trials

- baseline (unperturbed) on the generalization movements, 20 trials

- initial intermittent exposure to the force field, 100 trials

- initial intermittent exposure to generalization movements, 100 trials 


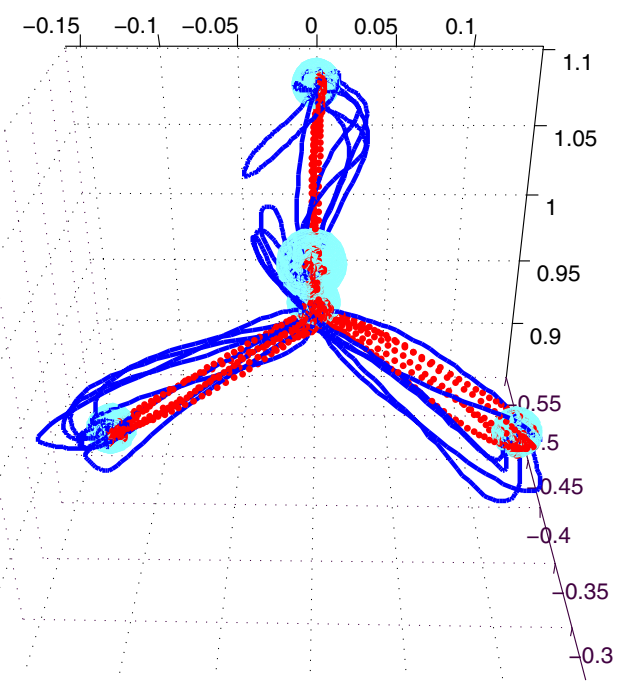

Fig. 4. Unconstrained baseline and after-effect movements using a $2.5 \mathrm{~N}$ viscous curl field. The blue lines indicate baseline movements, and the red lines indicate after-effect movements.

- training with force field, 300 trials

- after-effects 'catch trials': intermittent removal of the force field, 100 trials

- test on the generalization movements, 20 trials

- washout of the after-effects, 60 trials

The generalization trials were on 4 new and unpracticed movement directions. These directions were the vertices of another tetrahedron formed by taking the vertices of the 3D group and reflecting them about the horizontal plane.

During trials, VROOM collected data including the time stamps, current position, current orientation, and the current reaction force from the device. The system sampled these values at approximately $100 \mathrm{~Hz}$ intervals. The subjects head was held stationary using a chin rest and consequently magnetic tracking of the head was disabled for this experiment.

\section{B. Analysis Procedures}

Movements were analyzed based on phase and direction. Intermittent initial exposure and catch trials were isolated. Subjects' movement evaluations were based on five calculated error measures. The first error measure was the amount of time it took the subject to reach the target's destination. The second error was the trajectory path length from the starting point to the target. The ideal trajectory was considered a straight line between the two points. The next error measure was the initial direction, expressed as an angle relative to the direction of the target from the start position. The fourth error measure was the trajectory's perpendicular distance from the ideal linear trajectory. Finally, the fifth error measure was the depth component of the position error. Analysis concentrated on the perpendicular distance as the straightforward error measure for evaluating these experiment trials.

Fig 4 presents one subject's unconstrained movements toward the four targets. The blue lines indicate the 3D baseline trajectories without an active curl field, while the red lines indicate the 3D after-effect trajectories following exposure to the curl field. The figure has been rotated slightly for illustration purposes, but it is approximately the perspective viewed by subjects sitting at VRROOM. The closest target is between the center and the subject's head.

\section{RESULTS}

Examining the experiment results, we found that different error measures exhibited significance between the two groups of subjects' baseline and after-effect movements. For constrained 2D subjects, only the initial direction errors (paired t-test of baseline and after-effect error means,

$=0007351$ ) and perpendicular distance errors (paired t-test of error means, $\quad=0010624$ ) exhibited significant differences between baseline and after-effect movements. For unconstrained 3D subjects, only the time error (paired t-test of baseline and after-effect error means, $\quad=0$ 038748) and initial direction error (paired t-test of error means, = 0 030551) exhibited significant difference between baseline and after-effect movements. Due to previous experience, perpendicular distance was used as the primary error measure for comparing the subject groups. Fig. 5 illustrates the comparison between the constrained and unconstrained subjects' baseline and after-effect movements. The horizontal lines connect each subject's baseline and after-effect perpendicular distance error means.

We found that unconstrained 3D movements exhibited larger perpendicular distance errors than the constrained 2D planar movements (two-sample t-test of 2D and 3D means, $\quad=00019)$. There was also more trial-to-trial variability for the unconstrained movements (two-sampled t-test of 2D and 3D standard deviations, $\quad=0$ 000135). The unconstrained subjects' baseline movements were worse than the constrained subjects' after-effect movements. One 3D subject's average error decreased between the baseline and after-effect phases. The exact reasons for this decrease are unknown, as there are no apparent outliers. This subject's results contrast with the data for all other subjects, both constrained and unconstrained.

The movement errors were larger for unconstrained movements than planar constrained movements, but they also exhibited more variability. Constrained subjects' movements exhibited significant after-effects when compared to baseline movements (paired t-test of error means, 0 0348). In contrast, unconstrained subjects did not exhibit significant after-effects (paired t-test of error means: 0 1074), thus the collected data does not demonstrate that these subjects learned the viscous curl field's effects. Rather, the 3D movements fail to show an effect, and we surmise that any measurable effect of learning is buried within the excessive variability. Additionally, an analysis of generalization movements is reserved for a later publication.

\section{DISCUSSION}

We compared the baseline and after-effect movements' perpendicular distance errors. The results compare constrained and unconstrained subjects' movement trajectories 


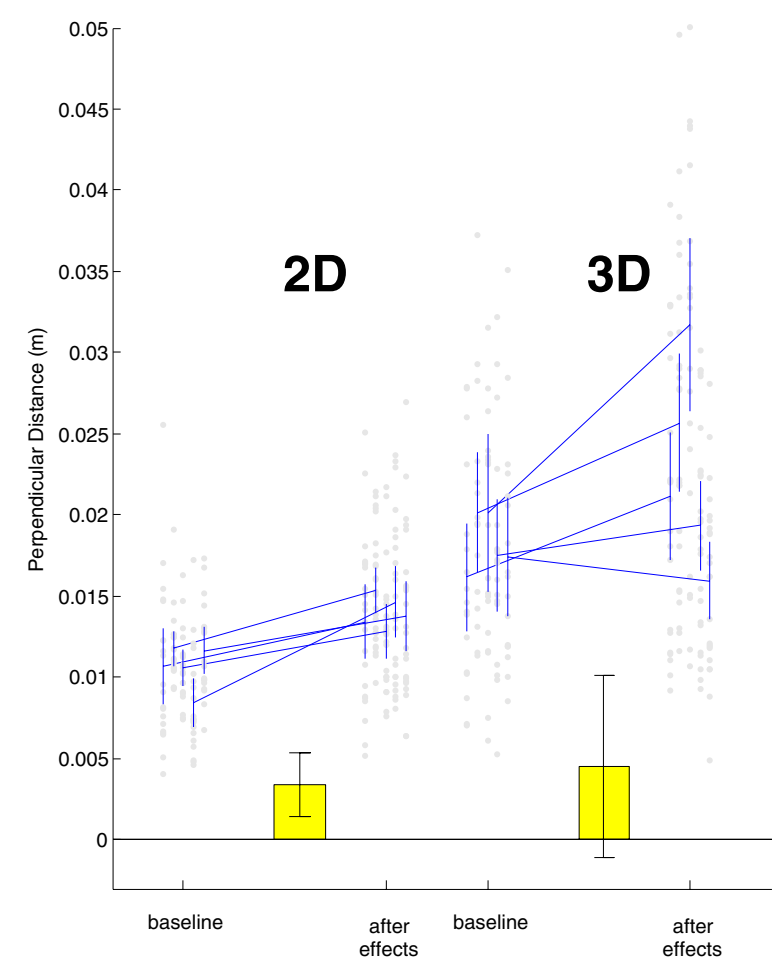

Fig. 5. Baseline and after-effect error changes for constrained planar 2D and unconstrained 3D movements. The bars indicate the average changes in error.

while experiencing a curl force field. Virtual reality appears to provide significant potential benefits medium for motor rehabilitation [26], [27], [18]. Haptics is an important addition [28]. The increased sense of presence resulting from sensory feedback has been shown to improve motivation to use the environment. Significant differences arise when moving existing research techniques into the virtual environment.

The larger trial-to-trial variability for unconstrained subjects' errors requires further investigation. Unconstrained subjects' movements exhibited larger trial-to-trial variability (standard deviation of perpendicular distance error, 0.0076 meters for baseline movements and 0.0088 meters for aftereffect movements) compared to the constrained 2D subjects' movements (standard deviation of perpendicular distance error, 0.0032 meters for baseline movements and 0.0045 meters for after-effect movements). The reasons for this variability among unconstrained subjects might be due to subjects having trouble correctly locating targets within the 3D workspace. Van den Dobbelsteen et. al. found that endpoint errors often resulted from judging endpoint positions [29]. Visual cues to depth are essential for properly determining the target positions within the workspace. VRROOM requires proper measurements of its screen position in order to calibrate the graphics display parameters, and any adjustments might cause larger errors. The magnetic tracking system was disabled during this experiment, thus removing motion parallax as a visual cue to depth.

There are two additional sources of challenge when going from 2 to 3 dimensions: gravity and an extra dimension. If the problem of gravity can be separated from the problem of three dimensions, then we can understand the nature of challenges facing the recovering neuromotor system after a stroke. Future experiments should differentiate the influence of gravity from the influence of added freedom of the hand. On one hand, the nervous system may have no trouble at all performing and adapting in a weighted, three-dimensional environment - this is, after all, a normal everyday condition. But on the other hand, larger contractions and hence noise may be present [30], and the nonlinear nature of gravitation may make it an excessively difficult process.

Another limitation in this experiment was the low intensity of the force field generated by the PHANToM. Although the pilot data displayed in Fig. 3 was collected using a $20 \mathrm{~N}$ force field, the experiments conducted for this paper reduced forces significantly to $2.5 \mathrm{~N}$. The PHANToM 3.0 exerts a maximum continuous force of only $1.75 \mathrm{~N}$. Resolving the problem of force magnitude necessitates using a robots capable of larger sustained forces. The laboratory has other robotic devices including the HapticMASTER from FCS Robotics [31] and the Whole Arm Manipulator (WAM) from Barret Technologies. These systems must be integrated with VRROOM, posing both physical and development challenges. Additional, modular software development must support these additional devices, both containing APIs very different from the GHOST SDK used to control the PHANToM.

Nevertheless our results show encouraging preliminary evidence that (1) the system developed can render the same conditions that previous planar systems did; (2) that free, three dimensional movements can be presented to an experimental subject; and interestingly; and (3) force field strengths that can elicit 2D constrained adaptation have a greater difficulty causing significant after-effects in $3 \mathrm{D}$. Future experiments with larger, stronger 3D robots will need to be in place to render the forces needed for significant neural adaptation that have been seen in two dimensions and that have the ability to bring about beneficial functional outcomes from robotic training.

\section{ACKNOWLEDGMENTS}

The authors gratefully acknowledge the contribution of Usha Periyanayagam and of VRCO, Inc. for providing development versions of CAVELib and trackd software. PARIS is a trademark of the Board of Trustees of the University of Illinois.

See http://www.smpp.northwestern.edu/RobotLab/ for more information on this and other research.

\section{REFERENCES}

[1] J. M. Hollerbach, "Dynamic interactions between limb segments during planar arm movements," Biological Cybernetics, vol. 44, pp. $67-77,1982$

[2] N. Hogan, "Mechanical impedance of single and multi-articular systems," in Multiple Muscle Systems, J. M. Winters and S. L.-Y. Woo, Eds. New York, New York: Springer-Verlag, 1990, pp. 149-164.

[3] F. E. Zajac, "Muscle and tendon: Properties, models, scaling, and application to biomechanics and motor control," CRC Critical Reviews in Bioengineering, vol. 17, pp. 359-411, 1989. 
[4] C. Ghez, "The control of movement," in Principles of Neural Science, E. R. Kandel, J. H. Scwartz, and T. M. Jessel, Eds., 1991, pp. 533-547.

[5] O. Bock, "Load compensation in human goal-directed arm movements," Behavioral Brain Research, vol. 41, pp. 167-177, 1990.

[6] J. R. Lackner and P. DiZio, "Rapid adaptation to coriolis force perturbations of arm trajectories," Journal of Neurophysiology, vol. 72, pp. 299-313, 1994.

[7] R. Shadmehr and F. A. Mussa-Ivaldi, "Adaptive representation of dynamics during learning of a motor task," Journal of Neuroscience, vol. 14, no. 5, pp. 3208-3224, 1994.

[8] F. Mussa-Ivaldi and J. L. Patton, "Robots can teach people how to move their arm," in IEEE International Conference on Robotics and Automation (ICRA), San Francisco, California, 2000.

[9] J. Patton, G. Dawe, C. Scharver, and R. Kenyon, "The development of a life-sized 3-d system for the rehabilitation of motor function," in Proceedings of 26th Annual International Conference of the IEEE Engineering in Medicine and Biology Society, Sep 52004.

[10] F. A. Miles and B. B. Eighmy, "Long -term adaptive changes in primate vestibuloocular reflex i: behavioral observations," Journal of Neurophysiology, vol. 43, pp. 1406-1425, 1980.

[11] J. R. Flanagan and A. K. Rao, "Trajectory adaptation to a nonlinear visuomotor transformation: evidence of motion planning in visually perceived space," Journal of Neurophysiology, vol. 74, no. 5, pp. 2174 8, 1995.

[12] H. Imamizu, S. Miyauchi, T. Tamada, Y. Sasaki, R. Takino, B. Putz, T. Yoshioka, and M. Kawato, "Human cerebellar activity reflecting an acquired internal model of a new tool. [see comments.]," Nature, vol. 403, no. 6766, pp. 192-5, 2000.

[13] J. W. Krakauer, Z. M. Pine, M. F. Ghilardi, and C. Ghez, "Learning of visuomotor transformations for vectorial planning of reaching trajectories," Journal of Neuroscience (Online), vol. 20, no. 23, pp. 8916-24, 2000.

[14] Y. Rossetti, G. Rode, L. Pisella, A. Farne, L. Li, D. Boisson, and M. T. Perenin, "Prism adaptation to a rightward optical deviation rehabilitates left hemispatial neglect," Nature, vol. 395, no. 6698, pp. 166-9, 1998.

[15] C. Tong, D. M. Wolpert, and J. R. Flanagan, "Kinematics and dynamics are not represented independently in motor working memory: evidence from an interference study," Journal of Neuroscience, vol. 22, no. 3 , pp. $1108-13,2002$

[16] Y. Wei and J. L. Patton, "Forces that supplement visuomotor learning: A 'sensory crossover' experiment," Experimental Brain Research, vol. conditionally accepted pending revisions, 2005.

[17] R. Azuma, "A survey of augmented reality," Presence: Teleoperators and Virtual Environments, vol. 6, no. 4, pp. 355-385, Aug 1997. [Online]. Available: http://www.cs.unc.edu/ $/$ azuma/ARpresence.pdf

[18] H. Sveistrup, "Motor rehabilitation using virtual reality," Journal of NeuroEngineering and Rehabilitation, vol. 1, no. 10, Dec 2004. [Online]. Available: http://bmc.ub.uni-potsdam.de/1743-0003-110/1743-0003-1-10.pdf

[19] C. Dean and R. Shepherd, "Task related training improves performance of seated reaching tasks after stroke: A randomize controlled trial,' Stroke, vol. 28, no. 4, pp. 722-728, 1997.

[20] A. Johnson, D. Sandin, G. Dawe, T. DeFanti, D. Pape, Z. Qiu, S. Thongrong, and D. Plepys, "Developing the paris: Using the cave to prototype a new vr display," in ACM Symposium on Immersive Projection Technology, Ames, Iowa, Jun 19-20 2000. [Online]. Available: http://www.evl.uic.edu/aej/papers/IPT.PARIS.pdf

[21] J. Salisbury and M. Srinivasan, "Phantom-based haptic interaction with virtual graphics," IEEE Computer Graphics and Applications, vol. 17, no. 5, pp. 6-10, 1997. [Online]. Available: http://www.computer.org/cga/cg1997/g5006abs.htm

[22] C. Scharver, R. Evenhouse, A. Johnson, and J. Leigh, "Pre-surgical cranial implant design using the paris prototype," in Proceedings of the IEEE Conference on Virtual Reality, Mar 27-31 2004, pp. 199-206.

[23] C. Cruz-Neira, D. Sandin, and T. DeFanti, "Virtual reality: The design and implementation of the cave," in Proceedings of the SIGGRAPH Computer Graphics Conference, Aug 1993, pp. 135-142. [Online] Available: http://www.evl.uic.edu/papers/pap_project.php3?indi=135

[24] P. Strauss and R. Carey, "An object-oriented 3d graphics toolkit," ACM SIGGRAPH Computer Graphics, vol. 26, no. 2, pp. 341-349, Ju 1992. [Online]. Available: http://doi.acm.org/10.1145/142920.134089

[25] SensAble Technologies, "Ghost sdk," 2001. [Online]. Available: http://www.sensable.com/

[26] A. Viau, A. Feldman, B. McFadyen, and M. Levin, "Reaching in reality and virtual reality: a comparison of movement kinematics in healthy subjects and in adults with hemiparesis," Journal of NeuroEngineering and Rehabilitation, vol. 1, no. 11, Dec 2004.

[27] J. Broeren, M. Rydmark, and K. Stibrant Sunnerhagen, "Virtual reality and haptics as a training device for movement rehabilitation after stroke: a single-case study," in Proceedings of the 4th International Conference on Disability, Virtual Reality, and Associated Technologies, Veszprem, Hungary, Sep 18-20 2002.

[28] D. Feygin, M. Kechner, and F. Teurlick, "Haptic guidance: Experimental evaluation of a haptic training method for a perceptual motor skill," in Proceedings of the 10th Symposium on Haptic Interfaces for Virtual Environment and Teleoperator Systems, Orlando, Florida, Mar 2002, pp. 40-47. [Online]. Available: http://itsa.ucsf.edu/ frankt/Feygin_Haptics_2002.pdf

[29] J. van den Dobbelsteen, E. Brenner, and J. Smeets, "Endpoints of arm movements to visual targets," Experimental Brain Research, no. 138, pp. 279-287, Apr 2001. [Online]. Available: http://www2.eur.nl/fgg/neuro/people/smeets/2001/endpointsEBR01.pdf

[30] C. M. Harris and D. M. Wolpert, "Signal-dependent noise determines motor planning," Nature, vol. 394, pp. 780-784, 1998. [Online]. Available: http://www.hera.ucl.ac.uk/papers/HarWol98.pdf

[31] R. van der Linde, P. Lammertse, E. Frederiksen, and B. Rulter, "The hapticmaster, a new high-performance haptic interface," in Proceedings of EuroHaptics 2002, Edinburgh, United Kingdom, Mar 2002. [Online]. Available: http://www.eurohaptics.vision.ee.ethz.ch/2002/vanderlinde.pdf 